\title{
A framework for risk management decisions in aviation safety at state level
}

\author{
D. Rios Insua ${ }^{\mathrm{a}}$, C. Alfaro $^{\mathrm{b}}, \mathrm{J}_{\text {. Gomez }}^{\mathrm{b}, *}$, P. Hernandez-Coronado ${ }^{\mathrm{c}}, \mathrm{F}$. Bernal $^{\mathrm{c}}$ \\ a ICMAT-CSIC, 28049 Madrid, Spain \\ b Rey Juan Carlos University, 28933 Móstoles, Spain \\ c Spanish Aviation Safety and Security Agency, 28020 Madrid, Spain
}

\section{A R T I C L E I N F O}

\section{MSC:}

\section{$00-01$}

99-00

Keywords:

Risk management

Aviation safety

Decision analysis

Bayesian statistics

\begin{abstract}
A B S T R A C T
Aviation is a key industrial sector for global development. Safety is essential for its healthy growth. However its management is pervaded by simplistic methods based on risk matrices. We provide here a framework for risk management decisions in aviation safety at state level. This helps us in identifying the best portfolio that a state agency may implement to improve aviation safety in a country. We illustrate our proposal with a case study.
\end{abstract}

\section{Introduction}

Organisations involved in aviation have been dealing with the prevention of accidents from the early days of this industry. Since the first aviation accident with casualties in 1908, many efforts have been spent in improving safety in the sector. After its creation in 1945, the International Civil Aviation Organization (ICAO) has focused interests in trying to make aviation the safest transportation mode. Statistics released by ICAO based on fatal accident rates support such efforts, with year 1968 marking a substantial improvement, see [1] for details. Since 2004, the accident rate has been relatively steady, with no significant improvement, averaging between 4 and 5 fatal accidents per 10 million flights. This could be due to the fact that aviation safety (AS) has reached a point in which safety benefits balance its costs, see [2] who consider that such point might have been reached in the late 1980s. However, an increasing deregulation and competition, as well as the expected increase in air traffic over the next decades, may put current safety levels into jeopardy.

The total elimination of aviation accidents and serious incidents is a desirable goal, but clearly unachievable. The idea of risk-free systems has evolved in recent years towards a perspective centered around safety management, aimed at supporting resource allocation processes in which a balance between "production" and "protection" is attained. In this context, [3] defines safety as the state in which the risk of harm to persons or property damage is reduced to, and maintained at or below, an acceptable level through a continuing process of hazard identification and risk management. AS management is articulated according to different levels and affects both the aviation service providers (airlines, airport operators, etc.) and the regulators of aeronautical services. This point of view is supported by ICAO through the regulatory framework of Safety Management Systems (SMS).

In this regard, it is worth noting that one of the most widespread methods for risk management in AS is based on risk matrices. A risk matrix is a tool for risk assessment and management that graphically represents the severity and likelihood of different risk factors [4], in our case called AS occurrences. Indeed, the most important regulatory organisations, such as ICAO, EASA, FAA or Eurocontrol, support and promote their use in all aviation sub-sectors, from airports to air traffic control, going through air navigation. Frequently, discrete scales of severity and probability values are used, whereby a table with cells associated with discrete levels in both magnitudes is defined, see [5]. The risk level of cells is represented with different colours (typically, red, yellow and green, which would suggest high, medium or low risk levels, respectively) facilitating risk visualisation. As an example, Table 1 represents the matrix recommended by ICAO [3].

We might think that this almost ubiquitous presence of risk matrices makes them a de facto standard. However, this methodology is criticised in the AS community, see e.g. [6,7]. A complete analysis of their weaknesses is available in [8], who considers that the use of low resolution $(5 \times 5$, for example) risk matrices with non-coherent colour schemes and subjective inputs can easily lead to erroneous risk management decisions. Such limitations suggest that risk matrices

\footnotetext{
* Corresponding author.

E-mail addresses: david.rios@icmat.es (D. Rios Insua), cesar.alfaro@urjc.es (C. Alfaro), javier.gomez@urjc.es (J. Gomez), phernandez@seguridadaerea.es (P. Hernandez-Coronado), fbernal@seguridadaerea.es (F. Bernal).
} 
Table 1

Risk matrix recommended by the ICAO.

\begin{tabular}{|l|c|c|c|c|c}
\hline \multirow{2}{*}{ Risk probability } & \multicolumn{5}{|c}{ Risk severity } \\
\cline { 2 - 7 } & Catastrophic & Hazardous & Major & Minor & Negligible \\
$\mathrm{A}$ & $\mathrm{B}$ & $\mathrm{C}$ & $\mathrm{D}$ & $\mathrm{E}$ \\
\hline Occasional 4 & $4 \mathrm{~A}$ & $4 \mathrm{~B}$ & $4 \mathrm{C}$ & $4 \mathrm{D}$ & $4 \mathrm{E}$ \\
\hline Remote 3 & $3 \mathrm{~A}$ & $3 \mathrm{~B}$ & $3 \mathrm{C}$ & $3 \mathrm{D}$ & $3 \mathrm{E}$ \\
\hline Improbable 2 & $2 \mathrm{~A}$ & $2 \mathrm{~B}$ & $2 \mathrm{C}$ & $2 \mathrm{D}$ & $2 \mathrm{E}$ \\
\hline Extremely improbable 1 & $1 \mathrm{~A}$ & $1 \mathrm{~B}$ & $1 \mathrm{C}$ & $1 \mathrm{D}$ & $1 \mathrm{E}$ \\
\hline
\end{tabular}

should be used with caution and only when careful explanations of the involved judgements can be provided. This is worsened by the fact that, as with our state level AS management problems, one needs to compare numerous occurrences of very different nature.

Netjasov and Janic [9] provide pointers to other approaches to risk and safety modelling in civil aviation including fault tree analysis, common cause analysis, event-tree analysis, bow-tie analysis and belief networks. In particular, [10] build a belief network to describe and predict causes of nine major occurrences. However none of the previous approaches properly integrate occurrence forecasting, however sophisticated their approaches are, with safety resource allocation.

In this paper, we propose a novel and systematic methodology for risk management in AS, based on the principles of decision and risk analysis. We begin by briefly introducing the proposed methodology. In Section 3, we detail its main steps: models to predict the occurrences and their severity classes; models to predict and assess occurrence consequences; risk maps to screen occurrences; and, finally, a procedure for safety management resource allocation. Section 4 illustrates the methodology with a case study, with masked data for confidentiality reasons. We conclude with a brief discussion.

\section{Framework}

\subsection{Introduction}

This section provides a framework to support a state in the identification of AS risks and the resource allocation to mitigate them. Despite the high safety level in the aviation industry, occurrences ${ }^{1}$ continue to emerge. Specifically, in our case, 88 different occurrence types will be considered, ranging from bird strike to runway excursion going through engine failure. Five occurrence classes are proposed by [3] depending on their severity: Accident (1); Serious Incident (2); Major Incident (3); Significant Incident (4); and Occurrence without safety effect (5). Thus, we may talk, for example, about an engine failure occurrence of class 3 .

Safety occurrences entail consequences. Each organisation must examine those of interest to them for risk management purposes. In our case, after a brainstorming process and a literature review, see in particular [11], the incumbent organisation (Spanish Aviation Safety and Security Agency, AESA) decided to focus on the following eight consequences identified as most relevant in AS management at state level $^{2}$ :

1. Fatalities associated with the functioning of the aviation system.

2. Minor wounded persons associated with the functioning of the system.

3. Severe wounded persons.

4. Delays caused by safety occurrences.

\footnotetext{
${ }^{1}$ ICAO defines "occurrence" to indicate an accident or an incident.

${ }^{2}$ For non-state actors in the aviation system, these could change and focus more on consequences related to profit and loss of the involved company. Similarly, for other countries, the selected consequences could be different.
}

5. Cancellations caused by safety occurrences.

6. Maintenance and repair operations produced by safety occurrences.

7. Destroyed aircrafts.

8. Image loss due to negative perception of occurrences.

All of the above consequences have natural attributes except the eighth one for which we used as proxy the number of accidents, as they are the occurrences that would tend to appear in the news.

As required by ICAO, each state must elaborate an AS plan which should lead to a resource allocation mechanism aimed at improving AS in the incumbent country. As usual in public policy, resources are limited and one must determine the best allocation taking into account various relevant constraints (economic, technical, logistic, legal, political, etc.). Thus, our aim is to establish a state-wide AS plan to minimise fatalities, injuries, induced delays and cancellations, the number of destroyed aircrafts, repair costs and, finally, the entailed image impact.

Given the current configuration of the aviation system, and taking into account the current AS state, a change in the resources allocated to different types of occurrences may have a global impact over such state and, therefore, possibly on the distribution of the occurrence rates, hoping to make them smaller and, therefore, make occurrences less frequent; and/or the proportions of occurrence classes, in an attempt to make the more severe occurrences less likely; and/or the consequences, reducing the associated impacts, if these were to occur. These are evaluated with the loss associated with that AS performance and, overall, with the expected loss of the corresponding safety policy. We shall try to minimise such expected loss, see [12].

\subsection{Model}

The problem we face is illustrated with the generic influence diagram in Fig. 1, where as usual, see [13], rectangular nodes represent decisions; the hexagonal node is a value node; circle nodes represent uncertainties; and, finally, doubly circular nodes represent deterministic nodes.

Here, $n$ designates the number of operations over the incumbent planning period and $k$ is the number of occurrences considered; $\lambda_{j}$ represents the rate of the $j$-th occurrence; $x_{j}$ is the number of $j$-th occurrences; $p_{j}=\left(p_{j}^{1}, \ldots, p_{j}^{5}\right)$ and $s_{j}=\left(s_{j}^{1}, \ldots, s_{j}^{5}\right)$ represent vectors that designate, respectively, the proportions and numbers of $j$-th occurrences at each severity class; $l_{j}$ designates the loss associated with the $j$ th occurrence; and, finally, loss represents the global loss.

We associate with each safety policy a portfolio of countermeasures $z=\left(z_{1}, z_{2}, \ldots, z_{k}\right)$, where $z_{j}$ will represent the proportion of resources (inspection time, personnel, investment, etc.) allocated to the $j$-th type of occurrence. To simplify the discussion we shall assume that there is a single type of resource. Then, the rate $\lambda_{j}$ of the $j$-th occurrence will follow a distribution $f\left(\lambda_{j} \mid z\right)=f\left(\lambda_{j} \mid z_{j}\right)$; and the split into the five occurrence classes $p_{j}=\left(p_{j}^{1}, \ldots, p_{j}^{5}\right)$ will follow a distribution $f\left(p_{j} \mid z\right)=f\left(p_{j} \mid z_{j}\right)$. Note that the quantity $z_{j}$ could have influence over $\lambda_{i}, i \neq j$, when the $i$-th and $j$-th occurrences are correlated, either due to a common antecesor, or because one of the occurrences typically precedes the other. However, we shall ignore such common effects here.

Once the plan $z$ is implemented, and given the number $n$ of operations:

- $x_{j}$ occurrences of the $j$-th type will emerge, split into $\left(s_{j}^{1}, s_{j}^{2}, s_{j}^{3}, s_{j}^{4}, s_{j}^{5}\right)$ occurrences in the five classes, with $x_{j}=\sum_{i=1}^{5} s_{j}^{i}$.

- The $g$-th occurrence of type $j$, designated $g_{j}$, results in $n_{F}^{g_{j}}$ fatalities, with distribution $f\left(n_{F} \mid j, z_{j}\right) ; n_{H}^{1 g_{j}}$ and $n_{H}^{2 g_{j}}$ minor and serious injured, with distribution $f\left(n_{H}^{1}, n_{H}^{2} l j, z_{j}\right) ; t_{D}^{g_{j}}$ accumulated delay, with distribution $f\left(t_{D} \mid j, z_{j}\right) ; n_{C}^{g_{j}}$ cancellations, with distribution $f\left(n_{C} \mid j, z_{j}\right)$; and, finally, $n_{R M}^{2 g_{j}}, \quad n_{R M}^{3 g_{j}}$ destructions or repairs, with distribution 


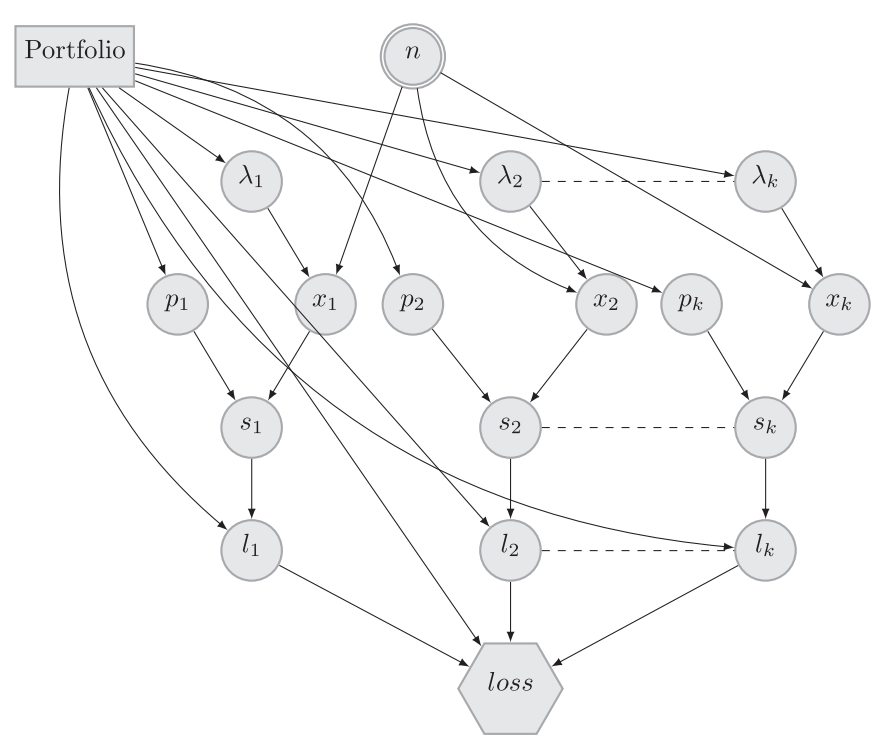

Fig. 1. Influence diagram for risk management in AS.

$$
f\left(n_{R M}^{2}, n_{R M}^{3} \mid j, z_{j}\right) \text {. }
$$

- Overall, these lead to $n_{\boldsymbol{F}}=\sum_{j=1}^{k} \sum_{g=1}^{x_{j}} n_{F}^{g_{j}}$ fatalities; $n_{\boldsymbol{H i}}=\sum_{j=1}^{k} \sum_{g=1}^{x_{j}} n_{H}^{i g_{j}}$, $i=1,2$, minor and serious injured, respectively; $t_{D}=\sum_{j=1}^{k} \sum_{g=1}^{x_{j}} t_{D}^{g_{j}}$, accumulated delay; $n_{C}=\sum_{j=1}^{k} \sum_{g=1}^{x_{j}} n_{C}^{g_{j}}$, cancellations; $n_{D}=\sum_{j=1}^{k} \sum_{g=1}^{x_{j}} n_{R M}^{2 g_{j}}$, destructions; $n_{R}=\sum_{j=1}^{k} \sum_{g=1}^{x_{j}} n_{R M}^{3 g_{j}}$, repairs; and, finally, $s^{1}=\sum_{j=1}^{k} s_{j}^{1}$, accidents.

- We would then evaluate these consequences with the loss function $l\left(n_{F},\left(n_{H 1}, n_{H 2}\right), t_{D}, n_{C},\left(n_{R}, n_{D}\right), s^{1}\right)$.

Then, for portfolio $z$, the corresponding expected loss $\psi(z)$ associated with the influence diagram in Fig. 1, would have the form

$\psi(z)=E\left(l\left(n_{F},\left(n_{H 1}, n_{H 2}\right), t_{D}, n_{C},\left(n_{R}, n_{D}\right), s^{1}\right) \mid z\right)$,

with the expectation defined with respect to the probability model in Fig. 1. Similarly, we could evaluate the expected loss contribution of the $j$-th occurrence type by limiting the consequences to just such occurrence.

\subsection{Risk maps for screening occurrences}

A first use of the above model allows us to screen the occurrences on which to focus the greatest AS risk management efforts. To avoid the problems associated with risk matrices mentioned above, we use risk maps in which the (continuous) $X$-axis refers to the likelihood of aviation occurrences and the (continuous) $Y$-axis conveys the severity of consequences associated with such occurrences. Specifically, here we shall represent the expected number of occurrences per 100,000 annual operations $(X)$ and the expected loss associated with such occurrence $(Y)$.

The idea of Pareto dominance is relevant here. Given a certain occurrence with associated coordinates $\left(x_{0}, y_{0}\right)$, Fig. 2 shows the $(x, y)$ locations of the better and worse occurrences with respect to such reference.

To wit, occurrences in the area $\left(x_{1}, y_{1}\right)$ are worse than the reference, since they tend to be more frequent and costly; occurrences $\left(x_{2}, y_{2}\right)$ are better, since they tend to be less frequent and costly; and, finally, occurrences $\left(x_{3}, y_{3}\right)$ and $\left(x_{4}, y_{4}\right)$ are incomparable with respect to $\left(x_{0}, y_{0}\right)$, since the first ones are more frequent but less costly, while the second ones are less frequent but more expensive. Then, occurrences on the "anti-Pareto" frontier of the risk map would require special attention, since there are no worse occurrences in the two relevant risk manage-

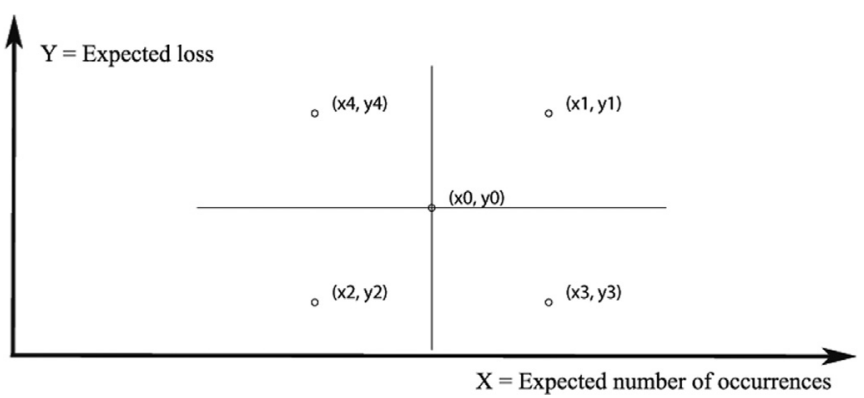

Fig. 2. Dominance in risk maps.

ment dimensions. Similarly, occurrences with higher expected losses or occurrence rates may seem worthy of attention since they are more costly and frequent, respectively.

In addition, risk maps from consecutive years are useful to identify occurrences that have worsened their risk level. For an occurrence characterised by $\left(x_{t}, y_{t}\right)$ in the $t$-th year map, such that $x_{t} \leq x_{t+1}, y_{t} \leq y_{t+1}$, it would seem to have worsened, as it tends to be more frequent and costly; if $x_{t} \geq x_{t+1}, y_{t} \geq y_{t+1}$, the occurrence seems to have improved since it tends to be less frequent and costly; whereas, finally, if $x_{t} \geq x_{t+1}$, $y_{t} \leq y_{t+1}$, or $x_{t} \leq x_{t+1}, y_{t} \geq y_{t+1}$, it would depend on how both criteria are aggregated.

Then, to screen AS occurrences on which to focus risk management, once the risk maps for years $(t-1)$ and $t$ are produced, respectively, called $\operatorname{map}_{t-1}$ and $\mathrm{map}_{t}$, we propose to (1) identify the occurrences in the anti-Pareto frontier of $\operatorname{map}_{t}$; (2) add some of the occurrences in map $_{t}$ that might produce higher losses; (3) add also some of the occurrences in map $_{t}$ that might be more frequent; (4) add those occurrences that worsened from $\operatorname{map}_{\mathrm{t}-1}$ to map $_{t}$; and (5) finally, include also novel occurrences emerging that year.

As mentioned, risk matrices are somewhat of a standard in AS. Therefore, we require a method to transform a risk map into a risk matrix for communication purposes with other aviation agencies. Based on the map, we draw cells to separate the occurrences using cutoff points for losses and frequencies proposed by the problem owner. We later adjust the levels so that they are equidistant, according to the definition of risk matrices, achieved through simple affine transformations. Finally, we specify the colours of cells, using the standard proposed by ICAO. We illustrate this in Section 4.5.

\subsection{AS resource allocation: stochastic version}

More importantly, we may provide a coherent safety resource allocation procedure based on the above elements. For simplicity, assume that only one type of resource is included, for example, based on inspection time, which is indeed the main resource available to the organisation at hand. Consider that a fraction $z_{j}$ of inspection time is allocated to address the $j$-th occurrence, with $z_{j} \geq 0, j=1, \ldots, k$, and $\sum_{j=1}^{k} z_{j}=1$. There may be additional constraints such as:

- Minimum inspection level for each occurrence: The organisation might require the inspection of at least a fraction $z_{\min } \geq 0$ to address each occurrence. This would be formulated as

$z_{j} \geq z_{\min }, \quad j=1, \ldots, k$.

- Maximum inspection level for each occurrence: The organisation could also establish a maximum level $z_{\max } \geq 0$ to address each occurrence. Formally, we represent this constraint through

$z_{j} \leq z_{\max }, \quad j=1, \ldots, k$.

Then, we associate with each policy $z$ its expected loss $\psi(z)$ as in (1) and aim at solving 
$\min \psi(z)$ s. t. $\sum_{j=1}^{k} z_{j}=1, z_{j} \geq z_{\min }, \quad j=1, \ldots, k, z_{j} \leq z_{\max }, \quad j=1, \ldots, k$.

The optimal solution would be $\left(z_{1}^{*}, \ldots, z_{k}^{*}\right)$, where $z_{j}^{*}$ would be the inspection time fraction allocated to address the $j$-th occurrence, $j=1, \ldots, k$. In order to compute the expected loss for a given policy, we would typically use a Monte Carlo approximation to (1) at a few portfolios and approximate its surface with a regression meta-model [14], optimising then $\widehat{\psi}(z)$ subject to the above constraints.

\subsection{Resource allocation: deterministic version}

The solution proposed in Section 2.4 may be expensive from a computational point of view. A more affordable approach would use a deterministic version of the risk management problem based on, for example, the expected values of the relevant random variables. To do this, given the inspection plan $z$, we define

- $E\left(\lambda^{j} \mid z\right)=\lambda_{z}^{j}$, the expected rate of the $j$-th occurrence.

- $E\left(X^{j} \mid z, n\right)=n \lambda_{z}^{j}$, the expected number of the $j$-th occurrences given $n$ operations.

- $E\left(p^{i j} \mid z\right)=p_{z}^{i j}$, the expected probability of occurrence severity class $i$ of the $j$-th occurrence, with $\sum_{i=1}^{5} p_{z}^{i j}=1, p_{z}^{i j} \geq 0, i=1, \ldots, 5$.

- $E\left(s_{j}^{i} \mid p^{i j}, z\right)=n \lambda_{z}^{j} p_{z}^{i j}$, the expected number of the $j$-th occurrences of severity class $i$ given $n$ operations.

- $E\left(r_{h}^{i j} \mid z\right)=m_{z h}^{i j}$, the expected value in the $h$-th consequence for a $j$-th occurrence of severity class $i, h=1, \ldots, 8$.

Then, $m_{h}^{z}=\sum_{j} \sum_{i} n \lambda_{z}^{j} p_{z}^{i j} m_{h z}^{i j}$ is the approximation of the expected value in the $h$-th consequence associated with inspection plan $z$. An approximation to the expected loss would be

$\widehat{\psi}(z)=l\left(m_{1}^{z}, \ldots, m_{8}^{z}\right)$.

The next step would be to solve the optimisation problem

$\min \widehat{\widehat{\psi}}(z)$ s. t. $\sum_{j=1}^{k} z_{j}=1, z_{j} \geq z_{\min }, \quad \forall j \in\{1, \ldots, k\}, z_{j} \leq z_{\max }$,

$\forall j \in\{1, \ldots, k\}$.

The optimal solution $\left(z_{1}^{*}, \ldots, z_{k}^{*}\right)$ would indicate the fraction of inspection time $z_{j}^{*}$ devoted to address the $j$-th occurrence, $j=1, \ldots, k$.

We discuss now how to model the influence of the inspection plan $z$ in the occurrence rates. To do this, we adopt the functional form

$\lambda_{z}^{j}=\delta^{j}+\exp \left(-\kappa^{j} z_{j}\right)$,

where $\delta^{j}$ and $\kappa^{j}$ are constants determined by solving the system

$\delta^{j}+\exp \left(-\kappa^{j} z^{j}\right)=\lambda^{j}, \delta^{j}+\exp \left(-\kappa^{j}\right)=\lambda^{j *}$.

Here, $\lambda^{j}$ designates the occurrence rate when the current resource level $z_{j}$ is invested and $\lambda^{j *}$ is the AS experts' estimation of the occurrence rate when the entire inspection resources are allocated to deal with the $j$-th occurrence, $z_{j}=1, j=1, \ldots, k$.

\section{Implementation}

We present some ideas about modelling the required elements in the nodes of the influence diagram in Fig. 1. For the implementation we have accessed numerous flight safety databases, mainly ECCAIRS; ${ }^{3}$ but also $\mathrm{ASN}^{4}$ and US DoT, ${ }^{5}$ among others.

\footnotetext{
${ }^{3}$ ECCAIRS is a software to support organisations in collecting, sharing, and analyzing their safety aviation information (http://eccairsportal.jrc.ec.europa.eu/).

${ }^{4}$ http://aviation-safety.net/

5 http://www.transtats.bts.gov/HomeDrillChart.asp
}

\subsection{Predicting the number of occurrences}

We outline the class of models used to predict the number of occurrences of each type in a given period, typically, a year or a month. Note that AS planning is performed annually, but monitoring is monthly and some of the occurrences present a seasonal (monthly) pattern. We focus on the case in which the occurrence rate is given as number of occurrences per 100,000 operations.

We use a model in which both the number of operations and the occurrence rate evolve dynamically, as in Fig. 3. For such purpose, we combine in a novel way several standard models. Specifically, we use a Dynamic Linear Model (DLM), see [15], to predict the number of operations (upper block); a Poisson model to predict the number of occurrences given the rate and number of operations (midblock); and, finally, a DLM to predict the evolution of the occurrence rate (lower block). With this class of models, we are able to deal with the effects we have found in the evolution of rates for all occurrence types, mainly the possible presence of seasonal and trend components.

The model in Fig. 3 is described through

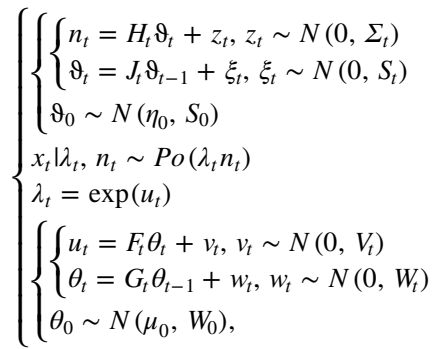

where, at month $t$,

- $n_{t}$ is the number of operations, which depends on a state variable $\vartheta_{t}$.

- $x_{t}$ is the number of occurrences of the relevant type, which depends on $\lambda_{t}$ and $n_{t}$ through a Poisson model.

- $\lambda_{t}$ defines the occurrence rate (number of occurrences per number of operations). For technical reasons, we define $\lambda_{t}=\exp \left(u_{t}\right)$, and $u_{t}$ evolves depending on a state variable $\theta_{\boldsymbol{t}}$.

- $F_{t}, G_{t}, H_{t}$, and $J_{t}$ would be matrices of a DLM, see [16].

- $v_{t}, w_{t}, z_{t}$, and $\xi_{t}$ would be independent sequences of normal variables with zero mean and variance matrices $V_{t}, W_{t}, \Sigma_{t}$, and $S_{t}$.

We provide an example in Section 4.1. To learn from data and predict

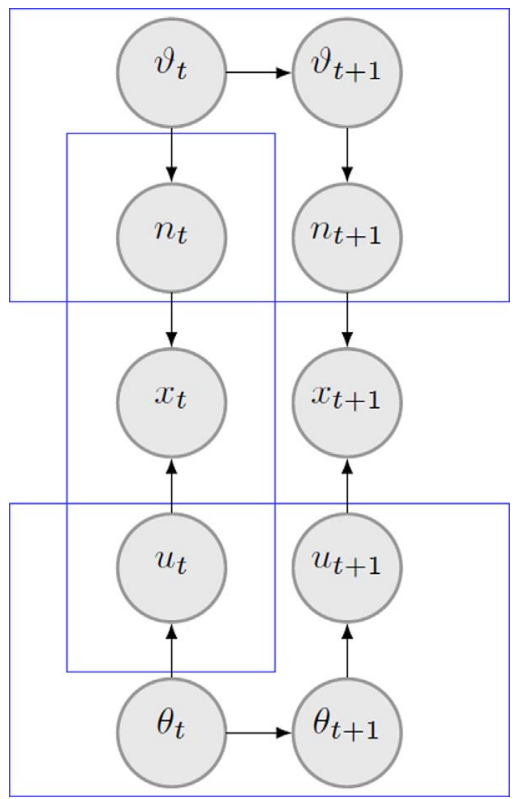

Fig. 3. Model to predict the number of occurrences. 
with these models we apply a particle filter, see [17]. Further details of these models may be seen in [18].

\subsection{Prediction of occurrence classes}

Conditional on the monthly number $x_{t}$ of occurrences, we must predict the corresponding numbers in occurrence classes which, as mentioned in Section 2, are five. Let $p=\left(p^{1}, p^{2}, p^{3}, p^{4}, p^{5}\right)$ be a vector designating the proportion of occurrences of each class with $p^{i} \geq 0$, $\sum_{i=1}^{5} p^{i}=1$. Let $s_{t}=\left(s_{t}^{1}, s_{t}^{2}, s_{t}^{3}, s_{t}^{4}, s_{t}^{5}\right)$ be a vector with the number of occurrences of each class with $s_{t}^{i} \geq 0$ and $\sum_{i=1}^{5} s_{t}^{i}=x_{t}$. Then, we use the multinomial-Dirichlet model.

$s_{t} \mid p, x_{t} \sim \mathcal{M}\left(x_{t} ; p^{1}, p^{2}, p^{3}, p^{4}, p^{5}\right), p \sim \mathcal{D i r}\left(\alpha_{1}, \alpha_{2}, \alpha_{3}, \alpha_{4}, \alpha_{5}\right)$.

Assuming that the data $D_{t}$ available until the beginning of the $t$-th period are $\left(\left(s_{1}{ }^{1}, s_{1}{ }^{2}, \ldots, s_{1}{ }^{5}\right), \ldots,\left(s_{t-1}^{1}, s_{t-1}^{2}, \ldots, s_{t-1}^{5}\right)\right)$, where $s_{j}{ }^{i}$ represents the number of occurrences of class $i, i \in\{1,2,3,4,5\}$, in period $j$, $j \in\{1, \ldots, t-1\}$, it is easily verified that, a posteriori, the distribution is Dirichlet with parameters

$p \mid D_{t} \sim \mathcal{D} i r\left(\alpha_{1}+\sum_{i=1}^{t-1} s_{i}^{1}, \ldots, \alpha_{5}+\sum_{i=1}^{t-1} s_{i}^{5}\right)$.

\subsection{Prediction of consequences}

We must predict also the eight consequences for the different types of occurrences and five severity classes. The kind of issues we need to address is, for example, assuming that there has been a bird strike occurrence of severity 2, forecast the number of minor injuries produced. In some cases, we shall need to make a distinction between the type of aircraft involved, for example, to predict more adequately, the number of fatalities. We use the following classification: T1, general aviation, aerial works, or business aviation, with less than 19 passengers; T2, regional flights ( $<100$ seats); T3, continental flights $(<200$ seats); and T4, intercontinental flights ( $>200$ seats). As an example, we sketch here the prediction of fatalities. For a full description for all relevant consequences, see [19].

\subsubsection{Prediction of fatalities}

Our aim is to build models to predict the number of fatalities for an AS occurrence for various types. From these models, by aggregation, we would obtain the distribution of the number of fatalities associated with a suggested AS management plan, including its segmentation according to occurrence type or aircraft type.

Several facts facilitate the construction of the model. First, there are only fatalities in occurrence class 1 , based on ICAO definition of accident. Furthermore, in an accident, there does not necessarily have to be fatalities, neither do all passengers and cabin crew have to die. Finally, the proportion of fatalities will typically depend on the type of aircraft and the type of occurrence.

The number $n_{F}$ of fatalities for an accident is predicted with a model

$n_{F}=p_{F} \cdot q \cdot M$,

where $p_{F}$ designates the proportion of fatalities; $q$, the aircraft occupancy degree; and $M$, its maximum occupancy. The first two parameters depend on the types of aircraft and occurrence. The third one just on the type of aircraft.

For the proportion $p_{F}$, we consider a mixture model

$p_{F} \sim \tau_{1} I_{0}+\tau_{2} \operatorname{Be}(a, b)+\tau_{3} I_{1}$,

where $\tau_{1}$ designates the proportion of accidents with no fatalities; $\tau_{2}$, the proportion of accidents in which there are both fatalities and survivors; and, finally, $\tau_{3}$, the proportion of accidents with no survivors.
We have $\tau_{1}+\tau_{2}+\tau_{3}=1, \tau_{i} \geq 0, i=1,2,3 . I_{0}$ is the degenerate distribution at 0 (no passenger dies); $\mathcal{B} e(a, b)$ models the distribution of the proportion of fatalities in accidents when there are fatalities and survivors; and finally, $I_{1}$ is the degenerate distribution at 1 (all passengers die).

To make inferences about weights $\tau_{i}$, we use a Dirichlet-multinominal model with posterior estimators $\hat{\tau}_{i}=\frac{a_{i}+s_{i^{\prime}}^{1}}{\sum_{i=1}^{3} a_{i}+s_{i^{\prime}}^{1}}$, where $a_{i}$ is the prior Dirichlet parameter and $s_{i}^{1}$ is the number of accidents in the $i$-th category for model (4), $i=1,2,3$. To perform inference over $p_{F}$, when $0<p_{F}<1$, we use a beta-binomial model with posterior estimators $\widehat{p}_{F}=\frac{a+\sum_{i=1}^{g} n_{F} \hat{i}}{a+b+\sum_{i=1}^{g} o_{i}}$, where $a, b$ are the prior beta parameters and $o_{i}, n_{F^{\prime}}{ }^{\prime} i$ are, respectively, the number of passengers and deaths in the $g$ accidents that led to some fatalities. For the occupancy proportion $q$, again, we use a beta-binomial model with posterior estimate $\widehat{q}=\frac{c+\sum_{i=1}^{f} p_{O_{i}}}{c+d+f}$, where $c$ and $d$ are prior beta parameters; $f$ is the number of flights for the period in question; and, finally, $p_{O_{i}}$ is the occupancy proportion of the $i$-th flight.

To estimate the cost associated with a fatality, we use the concept of value of statistical life (VSL), for example, presented in [20]. We use the reference value in [11] for Spain, which is $1.65 \mathrm{M€}$ and designate it $c_{F}$. Other estimations could be used, see [21] or [22] for details.

\subsection{Loss function}

We now describe the loss function used to assess an AS plan. We use the concepts of measurable multi-attribute value function [23] and relative risk aversion [24] to obtain a utility function. First, we aggregate the consequences through a measurable value function as

$$
\begin{aligned}
v\left(n_{F}, n_{H 1}, n_{H 2}, t_{D}, n_{C}, n_{R}, n_{D}, s^{1}\right)= & -c_{F} n_{F}-\sum_{i=1}^{2} c_{H i} n_{H i}-c_{D} t_{D}-c_{C} n_{C} \\
& -c_{R M}^{2} n_{D}-c_{R M}^{3} n_{R}-c_{I} s^{1},
\end{aligned}
$$

where $c_{F}=1.65 \mathrm{M} €$ is the cost associated with the loss of a human life; $c_{H 1}=0.43 \mathrm{M} €$ and $c_{H 2}=1.26 \mathrm{M} €$, estimated as proportions of the VSL, are the costs associated with minor and serious injuries, respectively; $c_{D}$ is the cost per minute of delay, approximated using a triangular distribution extracted from [11] (e.g, for a delay with network effect, it would be $c_{D} \sim \mathcal{T}(14.9,52.9,78.6)$ in $\left.€\right) ; c_{C}$ is the cost associated with the cancellation of a flight, depending on the aircraft type (e.g, for a type T4 aircraft, $\left.c_{C}=81,000 €\right) ; c_{R M}^{2}$ represents the cost associated with an aircraft destruction, which will depend on its type (e.g, for type T2, $\left.c_{R M}^{2}=80 \mathrm{M} €\right) ; c_{R M}^{3}$ designates the cost associated with an aircraft reparation, estimated from [8] using a triangular distribution for each aircraft type (e.g, for type T3, $c_{R M}^{3} \sim \mathcal{T}(306,671,1149)$ in $€$ ); and, lastly, $c_{I}=0.69 \mathrm{M} €$ is the image cost of each accident, elicited through expert judgement. Full details may be seen in [19]. The negative signs in the value function are due to the fact that we deal with costs to be minimised and value functions should be maximised, see [12].

We then assume that the regulator has constant absolute risk aversion with respect to $v$, see [25] for further details. Since this one is increasing, the utility function will be strategically equivalent to

$u(v)=-\exp (\omega v)$,

with $\omega<0$ designating the risk aversion coefficient, see [26] for further details. However, we prefer to adapt to the jargon in AS, and use loss functions (negative of utility functions) which, in our case, will be $l_{1}(v)=\exp (\omega v)$ as well as standardise it, giving a 0 loss to the best outcome and 1 to the worst one. We, then, formulate the system

$1-\alpha=\rho+\varrho \exp \left(\omega v_{1}\right)$,

$0=\rho+\varrho \exp \left(\omega v^{*}\right)$,

$1=\rho+\varrho \exp \left(\omega v_{*}\right)$,

where $\rho, \varrho$ and $\omega$ are constants to be determined; $v_{*}$ and $v^{*}$ are the 
Table 2

Priors parameters to predict the number of occurrences caused by bird strike.

\begin{tabular}{lllllllllllll}
\hline$\theta_{0}{ }^{L}$ & $\theta_{1}{ }^{L}$ & $\theta_{1}{ }^{S}$ & $\theta_{2}{ }^{S}$ & $\theta_{3}{ }^{S}$ & $\theta_{4}{ }^{S}$ & $\theta_{5}{ }^{S}$ & $\theta_{6}{ }^{S}$ & $\theta_{7}{ }^{S}$ & $\theta_{8}{ }^{S}$ & $\theta_{9}{ }^{S}$ & $\theta_{10}^{S}$ & $\theta_{11}^{S}$ \\
\hline 5 & 0.8 & 0 & 0 & 0 & 0 & 0 & 0 & 0 & 0 & 0 & 0 & 0
\end{tabular}

worst and best values attained, respectively; $v_{1}$ is an intermediate value between the worst and best consequences; and, finally, $\alpha$ is a value such that the lottery giving $v^{*}$ with probability $\alpha$ and $v_{*}$ with probability $(1-\alpha)$ leaves the AS manager indifferent with respect to obtaining $v_{1}$ with security.

\section{Case study}

We provide now an illustration of the proposed methodology. It is simplified because we consider only 24 of the total occurrences managed by the incumbent organisation and we consider only inspection resources.

\subsection{Prediction of the number of occurrences}

To illustrate the prediction of the number of occurrences, we consider the case of forecasting bird strikes using the model in Section 3.1. We have the monthly number of occurrences and operations from 2010 until 2014. An exploratory analysis suggests that its occurrence rate (number of bird strikes per 100,000 operations) shows seasonal (monthly) and linear growth effects. In this case, two state variables take care of the linear effect and eleven state variables refer to the monthly effect. Then, the $F_{i}$ vector is $((1,0),(1,0,0,0,0,0,0,0,0$, $0,0)$ ) and the $G_{i}$ matrix is

$\left[\begin{array}{ccccccccccccc}1 & 1 & 0 & 0 & 0 & 0 & 0 & 0 & 0 & 0 & 0 & 0 & 0 \\ 0 & 1 & 0 & 0 & 0 & 0 & 0 & 0 & 0 & 0 & 0 & 0 & 0 \\ 0 & 0 & -1 & -1 & -1 & -1 & -1 & -1 & -1 & -1 & -1 & -1 & -1 \\ 0 & 0 & 1 & 0 & 0 & 0 & 0 & 0 & 0 & 0 & 0 & 0 & 0 \\ 0 & 0 & 0 & 1 & 0 & 0 & 0 & 0 & 0 & 0 & 0 & 0 & 0 \\ 0 & 0 & 0 & 0 & 1 & 0 & 0 & 0 & 0 & 0 & 0 & 0 & 0 \\ 0 & 0 & 0 & 0 & 0 & 1 & 0 & 0 & 0 & 0 & 0 & 0 & 0 \\ 0 & 0 & 0 & 0 & 0 & 0 & 1 & 0 & 0 & 0 & 0 & 0 & 0 \\ 0 & 0 & 0 & 0 & 0 & 0 & 0 & 1 & 0 & 0 & 0 & 0 & 0 \\ 0 & 0 & 0 & 0 & 0 & 0 & 0 & 0 & 1 & 0 & 0 & 0 & 0 \\ 0 & 0 & 0 & 0 & 0 & 0 & 0 & 0 & 0 & 1 & 0 & 0 & 0 \\ 0 & 0 & 0 & 0 & 0 & 0 & 0 & 0 & 0 & 0 & 1 & 0 & 0 \\ 0 & 0 & 0 & 0 & 0 & 0 & 0 & 0 & 0 & 0 & 0 & 1 & 0\end{array}\right]$

Table 2 shows the prior estimates of the parameters in natural units, based on expert judgement [27], where $\theta_{0}{ }^{L}$ refers to the prior expected level, set at 5 occurrences; $\theta_{1}{ }^{L}$ refers to the expected growth, set at $\frac{10}{12}$ $(\approx 0.8)$ since we expect a growth of about 10 occurrences over a year (12 months). Finally, $\theta_{i}^{S}$ describes the $i$-th seasonal component, $i=1,2, \ldots,(12-1)$. Due to lack of information, we set them a priori to 0 . After applying a particle filter, see [17], the predictive mean and standard deviation (for the next month) were $m^{\prime}=84.7$ and $\sigma^{\prime}=29.3$, respectively. We proceed similarly for the other 23 occurrences.

\subsection{Prediction of occurrence classes}

We use expert judgement to obtain the prior parameters for a default prior for the split in occurrence classes in Section 3.2. The corresponding Dirichlet parameters were set at 1, 2, 3, 5 and 7 to indicate the greater probability of occurrence of classes 5, 4, 3, 2 and 1, respectively, but facilitating learning, by not adopting very high prior values. After processing the available data, the posterior parameters of the Dirichlet distribution for bird strike were $\operatorname{Dir}(1,3,77,3043,2998)$. As a consequence, for example, an estimate for the probability of a severity 3 bird strike would be $77 / 6132$.

We proceeded similarly for the 23 other occurrences.
Table 3

Posterior model parameters to forecast deaths due to bird strike accidents.

\begin{tabular}{lllllllllllll}
\hline Aircraft type & $s_{1^{\prime}}^{1}$ & $s_{2^{\prime}}^{1}$ & $s_{3^{\prime}}^{1}$ & $\hat{\tau}_{1}$ & $\hat{\tau}_{2}$ & $\hat{\tau}_{3}$ & $a$ & $b$ & $c$ & $d$ & $\hat{q}$ \\
\hline $\mathrm{T} 1$ & 18 & 2 & 4 & 0.70 & 0.11 & 0.19 & 8 & 5 & 10.52 & 15.48 & 0.40 \\
$\mathrm{~T} 2$ & 17 & 3 & 1 & 0.75 & 0.17 & 0.08 & 45 & 27 & 9.32 & 13.68 & 0.41 \\
$\mathrm{~T} 3$ & 7 & 2 & 0 & 0.67 & 0.25 & 0.08 & 37 & 184 & 7.88 & 3.12 & 0.72 \\
$\mathrm{~T} 4$ & 6 & 0 & 1 & 0.7 & 0.1 & 0.2 & 1 & 1 & 2.7 & 6.3 & 0.34 \\
\hline
\end{tabular}

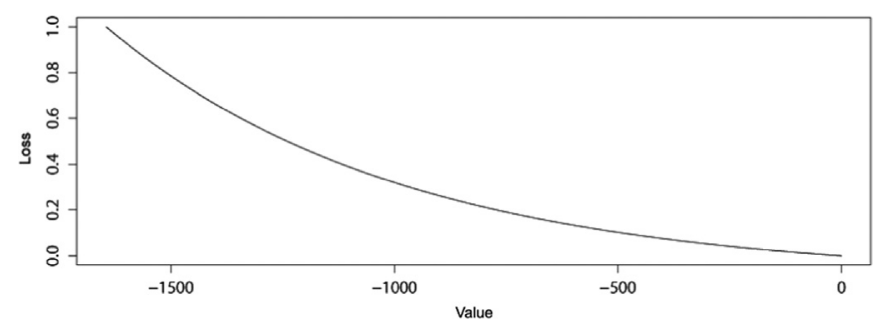

Fig. 4. Loss function.

\subsection{Prediction of number of deaths due to bird strike}

For fitting, we used data available from the Aviation Safety Network $^{6}$ which contains information of accidents worldwide since 1919. We web scrapped this information considering only data from 1968, the year in which a substantial improvement was achieved in AS, see [1], and included only civil aircraft accidents. In addition, we segmented the information depending on the type of aircraft involved, according to the T1-T4 classification suggested above.

Table 3 summarises posterior model parameters for bird strikes depending on aircraft type, stemming from non-informative priors, following the notation in Section 3.3.1.

We proceeded analogously for the other 23 types of occurrences and the other 7 types of consequences, as described in Section 3.3.1.

\subsection{Loss Function}

To build the loss function, we consulted with several experts from the organisation to obtain the values for each of the variables in (5), leading to system

$0.2=\rho+\varrho \exp (\omega \cdot(-771.52))$,

$0=\rho+\varrho \exp (\omega \cdot 0)$,

$1=\rho+\varrho \exp (\omega \cdot(-1644.94))$.

Here, $v^{*}=0$ refers to the best possible situation with no occurrences (and, therefore, no deaths, no injured persons,..., no image loss); $v_{*}=-1644.94$, to the worst possible situation taking as reference the worst values for each of the eight relevant consequences identified from the available data $\left(n_{F}=163, n_{H 2}=31, \ldots, s_{1}=44\right)$; and $v_{1}=-771.52$ refers to the value of an intermediate situation $\left(n_{F}=16, n_{H 2}=8, \ldots\right.$, $s_{1}=29$ ). An $\alpha$ value of 0.8 is elicited from AS experts. Solving system (6), we obtain $\widehat{\rho}=-0.09, \widehat{\varrho}=0.09$ and $\widehat{\omega}=-0.00151$. Fig. 4 represents the loss function.

\subsection{Risk maps and matrices}

We present in Fig. 5 the 2015 risk map for AS in Spain with the 24 incumbent occurrences. In parallel, we also provide the corresponding risk matrix. The context we consider is predictive, that is, we use data until 2014 to predict the 2015 map (and matrix).

To build the risk matrix we used $(10,20,40,60)$ as cutoff levels for the expected number of occurrences and $(0.005,0.01,0.035,0.055)$ for

\footnotetext{
${ }^{6}$ http://aviation-safety.net/
} 


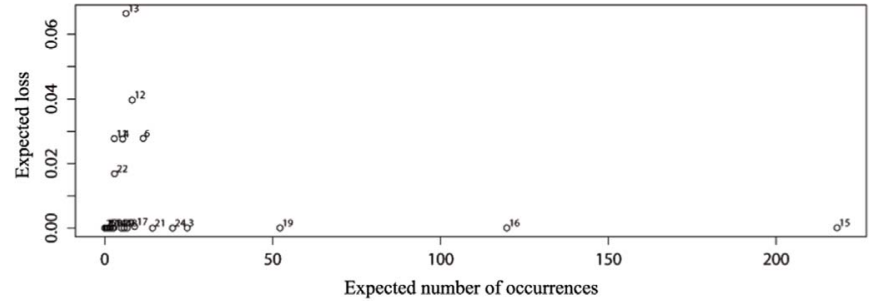

(a)

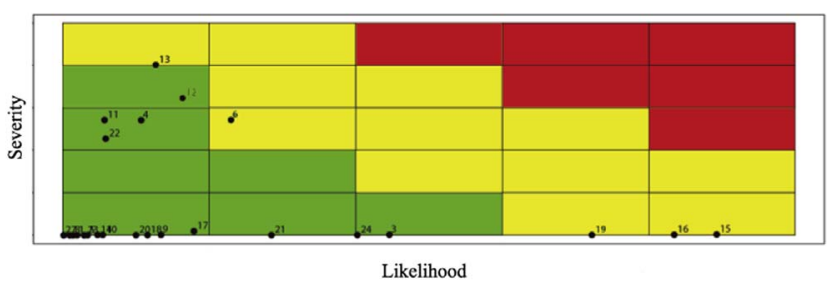

(b)

Fig. 5. (a) Risk map and (b) risk matrix for 2015 .

Table 4

Screened occurrences for 2015.

\begin{tabular}{lllll}
\hline Anti-Pareto & High loss & High frequency & Worse & Emerge \\
\hline \multirow{2}{*}{ ECO (13) } & ECO (13) & ATC (15) & TWS (4) & TWS (4) \\
& RE (6) & & RE (6) & \\
LAO (12) & TWS (4) & & CFIT & \\
ATC (15) & LAO (12) & LAO (12) & CFIT (11) \\
& CFIT (11) & ECO (13) & \\
RE (6) & FE (22) & FE (22) & \\
\hline
\end{tabular}

Table 5

Expected probabilities for occurrences.

\begin{tabular}{lllllll}
\hline ID & Occurrence type & $E\left(p^{1 j}\right)$ & $E\left(p^{2 j}\right)$ & $E\left(p^{3 j}\right)$ & $E\left(p^{4 j}\right)$ & $E\left(p^{5 j}\right)$ \\
\hline 1 & ECO & 0.07 & 0.04 & 0.11 & 0.4 & 0.38 \\
2 & ATC & 0.007 & 0.003 & 0.01 & 0.78 & 0.2 \\
3 & TWS & 0.09 & 0.12 & 0.28 & 0.32 & 0.19 \\
4 & RE & 0.09 & 0.27 & 0.22 & 0.33 & 0.09 \\
5 & CFIT & 0.43 & 0.17 & 0.1 & 0.07 & 0.23 \\
6 & LAO & 0.37 & 0.12 & 0.24 & 0.15 & 0.12 \\
7 & FE & 0.19 & 0.09 & 0.31 & 0.19 & 0.22 \\
\hline
\end{tabular}

Table 6

Expected forecasted fatalities (only relevant if severity is 1 ; in other cases it is 0 ); injuries (minor and serious) per occurrence.

\begin{tabular}{lllll}
\hline ID & $\begin{array}{l}\text { Occurrence type } \\
\text { Occur. Class }\end{array}$ & $\begin{array}{l}\text { Fatalities } \\
1\end{array}$ & $\begin{array}{l}\text { Minor Inj. } \\
1-4\end{array}$ & $\begin{array}{l}\text { Serious Inj. } \\
1-4\end{array}$ \\
\hline 1 & ECO & 2.94 & 0.002 & 0.0006 \\
2 & ATC & 0.0001 & 0.003 & 0.0007 \\
3 & TWS & 0.0002 & 0.0003 & 0.0008 \\
4 & RWE & 5.89 & 0.001 & 0.0004 \\
5 & CFIT & 24.5 & 0.00008 & 0.00004 \\
6 & LAO & 11.47 & 0.002 & 0.0005 \\
7 & FE & 7.12 & 0.000002 & 0.000001 \\
\hline
\end{tabular}

the expected loss.

\subsection{Screening occurrences}

Table 4 shows the list of occurrences over which to concentrate AS management efforts, if we apply the screening approach proposed in Section 2.3 for year 2015. Note that a same occurrence may appear in several of the categories. For example, ATC appears both in the anti-
Table 7

Delay associated (for accident 1, cancellation); expected probabilities of cancellation, repair and destruction (only relevant in accidents) per occurrence.

\begin{tabular}{|c|c|c|c|c|c|c|c|}
\hline \multirow[b]{2}{*}{ ID } & \multirow{2}{*}{$\begin{array}{l}\text { Occurrence type } \\
\text { Occurr. Class }\end{array}$} & \multicolumn{2}{|l|}{ Delay } & \multicolumn{2}{|c|}{ Cancell. } & \multirow{2}{*}{$\begin{array}{l}\text { Repair } \\
2-5\end{array}$} & \multirow{2}{*}{$\begin{array}{l}\text { Destruc. } \\
1\end{array}$} \\
\hline & & $2-3$ & $4-5$ & 1 & $2-5$ & & \\
\hline 1 & $\mathrm{ECO}$ & 10.29 & 0.29 & 1 & 0.02 & 0.14 & 0.17 \\
\hline 2 & ATC & 0.59 & 0.005 & 1 & 0.02 & 0.13 & 0.0002 \\
\hline 3 & TWS & 8.08 & 5.71 & 1 & 0.02 & 0.0001 & 0.0001 \\
\hline 4 & $\mathrm{RE}$ & 4.29 & 2.37 & 1 & 0.02 & 0.08 & 0.1 \\
\hline 5 & CFIT & 37.59 & 18.06 & 1 & 0.02 & 0.0001 & 0.42 \\
\hline 6 & LAO & 17.12 & 6.5 & 1 & 0.02 & 0.0002 & 0.37 \\
\hline 7 & $\mathrm{FE}$ & 38.71 & 20.21 & 1 & 0.02 & 0.32 & 0.36 \\
\hline
\end{tabular}

Table 8

Expected and parameter rates. Optimal inspection percentage for each occurrence.

\begin{tabular}{llllll}
\hline Occurrence type & Current rate & Min. rate & $\delta^{j}$ & $\kappa^{j}$ & \% Inspec. \\
\hline ECO & 0.37 & 0.02 & 0.02 & 7.29 & 15 \\
ATC & 11.57 & 0.21 & 5.48 & 2.27 & 5 \\
TWS & 0.27 & 0.01 & 0.01 & 9.5 & 5 \\
RE & 0.52 & 0.03 & 0.02 & 4.8 & 5 \\
CFIT & 0.13 & 0.007 & 0.007 & 14.67 & 21 \\
LAO & 0.44 & 0.02 & 0.019 & 6.07 & 30 \\
FE & 0.15 & 0.008 & 0.008 & 13.66 & 19 \\
\hline
\end{tabular}

Pareto frontier and because of its higher predicted frequency.

\subsection{Resource allocation}

We provide an illustration of the resource allocation process described in Section 2.5, in which we assume that we include the $k=7$ occurrences in Table 4. The assumed context is that we are finishing 2014 and must determine an inspection plan for 2015. Assume that, currently, we are inspecting the 7 occurrences equally intensely, that is $z_{j}=\frac{1}{7}, j=1, \ldots, 7$. We want to study whether there is a better inspection plan. We show the required data for the seven involved occurrences.

We first indicate in Table 5 the expected proportions for each severity class and type of occurrence, under the model in Section 4.2. Note that CFIT and LAO seem more prone to leading to accidents, whereas ECO and ATC seem the least dangerous occurrences.

We then display in Table 6 (fatalities, injuries) and Table 7 (delays, cancellations, repairs, destructions) the expected consequences for each occurrence class and type of occurrence. Recall that a generic model to forecasting fatalities was described in Section 3.3.1. CFIT and LAO accidents seem to lead to more fatalities.

CFIT and FE lead to bigger delays. CFIT, LAO and FE have a much higher expected probability of destruction. FE has a much higher expected probability of repair.

Finally, Table 8 shows the current expected rates $\left(z_{j}=\frac{1}{7}\right)$ and the minimum achievable, when all inspection resources are dedicated to the corresponding occurrence (i.e., $z_{j}=1$ ), as assessed by an expert.

We adjust the rates of different occurrences to the inspection level. We fit the model as described in Section 2.5. For example, for the $E C O$ occurrence (to which we allocate $z_{1}$ ) we have

$\delta^{1}+\exp \left(-\kappa^{1} \frac{1}{7}\right)=0.37, \delta^{1}+\exp \left(-\kappa^{1}\right)=0.02$.

This system leads to $\delta^{1}=0.02$ and $\kappa^{1}=7.29$. Table 8 summarises the parameters for various occurrences.

We describe now how to estimate the associated consequences to the inspection plan $z=\left(z_{1}, z_{2}, z_{3}, z_{4}, z_{5}, z_{6}, z_{7}\right)$, focusing on the $E C O$ occurrence:

The rate is $\delta^{1}+\exp \left(-\kappa^{1} z_{1}\right)=\lambda\left(z_{1}\right)$. The number of expected occur- 
rences is $n \lambda\left(z_{1}\right)$, which we designate $x\left(z_{1}\right)$. Additionally, the expected number of occurrences in the five classes is $\left(0.07 x\left(z_{1}\right), 0.04 x\left(z_{1}\right)\right.$, $\left.0.11 x\left(z_{1}\right), 0.4 x\left(z_{1}\right), 0.38 x\left(z_{1}\right)\right)=\left(s^{1}\left(z_{1}\right), s^{2}\left(z_{1}\right), s^{3}\left(z_{1}\right), s^{4}\left(z_{1}\right), s^{5}\left(z_{1}\right)\right)$.

- The expected number of fatalities is $m_{1}\left(z_{1}\right)=2.94 s^{1}\left(z_{1}\right)$.

- The expected number of minor injuries is $m_{2}\left(z_{1}\right)=0.002 \sum_{i=1}^{4} s^{i}\left(z_{1}\right)$.

- The expected number of serious injuries, $m_{3}\left(z_{1}\right)=0.0006 \sum_{i=1}^{4} s^{i}\left(z_{1}\right)$.

- The induced expected time delay will be $m_{4}\left(z_{1}\right)=10.29\left(s^{2}\left(z_{1}\right)+s^{3}\left(z_{1}\right)\right)+0.29\left(s^{4}\left(z_{1}\right)+s^{5}\left(z_{1}\right)\right)$.

- The number of expected cancellations would be $m_{5}\left(z_{1}\right)=s^{1}\left(z_{1}\right)+0.02\left(s^{2}\left(z_{1}\right)+s^{3}\left(z_{1}\right)+s^{4}\left(z_{1}\right)+s^{5}\left(z_{1}\right)\right)$.

- The expected number of damaged aircrafts would be $m_{6}\left(z_{1}\right)=0.14\left(s^{2}\left(z_{1}\right)+s^{3}\left(z_{1}\right)+s^{4}\left(z_{1}\right)+s^{5}\left(z_{1}\right)\right)$.

- The expected number of destroyed aircrafts is $m_{7}\left(z_{1}\right)=0.17 s^{1}\left(z_{1}\right)$.

- The expected number of accidents is $m_{8}\left(z_{1}\right)=0.07 s^{1}\left(z_{1}\right)$.

This would be carried out for the other types of occurrences similarly, resulting in the following overall consequences associated with plan $z$ :

$m_{h}(z)=\sum_{j=1}^{7} m_{h}\left(z_{j}\right), \quad h=1, \ldots, 8$.

Then, the value associated with the inspection plan $z$ would be

$$
\begin{aligned}
v(z)= & -c_{F} m_{1}(z)-c_{H 1} m_{2}(z)-c_{H 2} m_{3}(z)-c_{D} m_{4}(z)-c_{C} m_{5}(z)-c_{R M}^{2} m_{6}(z) \\
& -c_{R M}^{3} m_{7}(z)-c_{I} m_{8}(z) .
\end{aligned}
$$

Since $u$ is an increasing monotonic function, optimizing $u(v(z))$ is equivalent to optimizing $v(z)$. Then, if we want to, for example, ensure a minimum inspection level per occurrence, say 0.05 , and a maximum level, say 0.3 , we would solve the problem

$\max v(z)$ s. t. $\sum_{j=1}^{7} z_{j}=1,0.05 \leq z_{j} \leq 0.3, \quad i=1, \ldots, 7$.

The optimal solution is $z_{1}^{*}=0.15, z_{2}^{*}=0.05, z_{3}^{*}=0.05, z_{4}^{*}=0.05$, $z_{5}^{*}=0.21, z_{6}^{*}=0.3, z_{7}^{*}=0.19$, displayed as percentages in the last column of Table 8 .

\section{Discussion}

In striking contrast with the technological sophistication achieved in the aviation system from the aeronautical engineering perspective, risk management in AS is pervaded by unsophisticated methods evolving around the concept of risk matrix see [28,29], with its potential pitfalls.

We have proposed a methodology for risk management in AS based on sound principles of risk and decision analysis [30]. Its main advantages are providing an integrated coherent framework for safety resource allocation taking advantage of all available information, both from data and expert judgment. We also support risk monitoring, reporting and screening. We present two versions of the general model, stochastic and deterministic, to be implemented depending on the level of accuracy required and the available computational resources. The methodology is useful in defining the countermeasures that allow us to manage the resources referred to in [31], minimizing the risks associated with AS, taking into account various constraints (economic, technical, logistic, etc.) over such resources. We have illustrated the methodology with a simplified example.

On the other hand, the approach is much more technical and sophisticated than the above-mentioned risk matrix based methods. We have countered this partly by training engineers in charge of implementing in practice the methodology, partly by developing
RIMAS, a decision support system implementation of the proposed methodology. Beyond these, future work includes improving the occurrence forecasting methodology with the aid of SGDLMs from [32]; monitoring the implementation of the methodology to evaluate its actual impact in AS and eventually improve it and RIMAS; and, finally, extending it to include data from Flight Data Monitoring systems to improve occurrence predictions. Moreover, for the most worrisome occurrences we should undertake detailed studies, possibly through causal networks as in [10].

\section{Acknowledgement}

This work is supported by an AESA-RAC agreement, the MINECO MTM2014-56949-C3-1-R project and the AXA-ICMAT Chair in Adversarial Risk Analysis. The authors are grateful to the support of the COST IS1304 Action on Expert Judgement. Finally, we thank the referees for various suggestions that improved the paper.

\section{References}

[1] EASA. Annual safety review 2010. European Aviation Safety Agency (EASA); Cologne. 2011

[2] Vasigh B, Tacker T, Fleming K. Introduction to air transport economics: from theory to applications. Aldershot: Ashgate Publishing, Ltd.; 2008.

[3] ICAO. Safety management manual (Doc 9859 AN/474). International Civil Aviation Organization (ICAO); Montreal. 2006.

[4] Ni CA, H, Chen N. Some extensions on risk matrix approach. Saf Sci 48(10);2010:1269-78

[5] Ayres Jr M, Shirazi H, Cardoso S, Brown J, Speir R, Selezneva OI, et al. Safety management systems for airports. Volume 2: Guidebook; 2009.

[6] Cioacă C. Qualitative risk analysis methods in aviation projects. J Def Resour Manag 2011;01:77-84.

[7] Edwards C, Westcott M, Fulton N. The application of hazard risk assessment in defence safety standards. In: Proceedings of improving systems and software engineering conference; 2009. p. 135-46.

[8] Cox L. What's wrong with risk matrices?. Risk Anal 2008;28(2):497-512.

[9] Netjasov F, Janic M. A review of research on risk and safety modelling in civil aviation. J Air Transp Manag 2008;14(4):213-20.

[10] Ale BJ, Bellamy L, Van der Boom R, Cooper J, Cooke RM, Goossens LH, et al. Further development of a causal model for air transport safety (CATS): building the mathematical heart. Reliab Eng Syst Saf 2009;94(9):1433-41.

[11] EUROCONTROL. Standard inputs for eurocontrol cost benefit analyses. Technical report, Technical report 6. European Organisation for the Safety of Air Navigation (EUROCONTROL); Septiembre Brussels. 2013

[12] French S, Ríos Insua D. Statistical decision theory. London: Arnold Publishers; 2000.

[13] Banks D, Rios J, Rios Insua D. Adversarial risk analysis, vol. 343. Florida: CRC Press; 2015.

[14] Kleijnen JP, Sargent RG. A methodology for fitting and validating metamodels in simulation. Eur J Oper Res 2000;120(1):14-29.

[15] Harrison J, West M. Bayesian forecasting \& dynamic models. New York: Springer; 1999.

[16] Petris G, Petrone S, Campagnoli P. Dynamic linear models with R. New York: Springer; 2009.

[17] Rios Insua D, Ruggeri F, Wiper M. Bayesian analysis of stochastic process models, vol. 978. New York: John Wiley \& Sons; 2012.

[18] Rios Insua D, Alfaro C, Gomez J, Hernandez-Coronado P, Bernal F. A class of forecasting models for aviation safety. Technical report; 2016.

[19] Rios Insua D, Alfaro C, Gomez J, Hernandez-Coronado P, Bernal F. Forecasting and assessing consequences in aviation safety. Technical report; 2016.

[20] Viscusi WK, Aldy JE. The value of a statistical life: a critical review of market estimates throughout the world. J Risk Uncertain 2003;27(1):5-76.

[21] Thaler R, Rosen S. The value of saving a life: evidence from the labor market. In: Household production and consumption. NBER; Cambridge. 1976. p. 265-302.

[22] Miller TR. Variations between countries in values of statistical life. J Transp Econ Policy 2000:169-88.

[23] Dyer JS, Sarin RK. Measurable multiattribute value functions. Oper Res 1979;27(4):810-22

[24] Dyer JS, Sarin RK. Relative risk aversion. Manag Sci 1982;28(8):875-86.

[25] Ortega J, Ortega V, Rios Insua D. Utility elicitation. In: Días L, Morton A, Quigley J, editors. Handbook of judgment elicitation.. Springer; 2016.

[26] Keeney RL, Raiffa H. Decisions with multiple objectives: preferences and value trade-offs. New York: Cambridge University Press; 1993.

[27] Cooke RM, Goossens LHJ. Expert judgement elicitation for risk assessments of critical infrastructures. J Risk Res 2004;7(6):643-56.

[28] ICAO. Safety management manual (Doc 9859 AN/474). International Civil Aviation 
Organization (ICAO); Montreal. 2013.

[29] FAA. A introduction to safety management systems (SMS) for airport operators, no. : Ac 150/5200-37. Technical report, Federal Aviation Administration (FAA); Washington. 2007.

[30] Von Winterfeldt D, Edwards W. Decision analysis and behavioral research, vol.
604. Cambridge: Cambridge University Press; 1986.

[31] EASA. European aviation safety plan 2014-2017. European Aviation Safety Agency (EASA); Brussels. 2013.

[32] Gruber LF, West M. Bayesian forecasting and portfolio decisions using simultaneous graphical dynamic linear models; 2015. 